\title{
DIAGNOSTIC ACCURACY OF FINE NEEDLE ASPIRATION CYTOLOGY FOR PALATAL MINOR SALIVARY GLAND TUMORS USING DIFFERENT NEEDLE MATERIALS
}

\author{
Mohamoud Elsayed Khalifa*, Ahmed Abdelaziz Mohamed Essa** and Samy M. El-Safty ${ }^{* * *}$
}

\begin{abstract}
Background: Fine-needle aspiration cytology (FNAC) has been considered as an initial diagnostic tool for any swelling in major salivary glands and some minor salivary glands. It has been used by surgeons as a clinical tool to differentiate between benign and malignant tumors of the salivary glands. Superficial location of palatal minor salivary glands makes them most favorable target for FNAC. The surgical intervention is planned depending on the results of cytology.
\end{abstract}

Aims: To establish correlation between cytological and histopathological diagnoses of palatal salivary gland lesions and to examine accuracy of aspiration cytology as an early diagnostic aid using different needle materials (Cobalt Chromium and Stainless Steel).

Materials and Methods: Sixty patients presenting with palpable palatal lesions were included over a period from September 2015 to September 2017. FNAC was carried out for all cases and a correlation was conducted between clinical, cytological and histopathological outcomes. Patients were divided into two main groups $(n=30)$; in Group A, the aspiration was done using a CobaltChromium "Co-Cr" needle, attached to a syringe, while in Group B, it was performed using a stainless steel needle.

Results: The results of the FNAC specimens obtained from the 60 palatal lesions were correlated to their histopathological findings. FNAC results - supported by the histopathological investigations - revealed 45 pleomorphic adenomas, 5 mucoepidermoid carcinomas, 2 adenoid cystic carcinomas, 5 squamous cell carcinomas, 2 nonspecific inflammations and one case represented normal salivary gland tissue. With regard to complications of the fine needle aspiration procedure in this study, Co-Cr needles proved better biocompatibility and lower deformity in cells collected than Stainless steel needles.

Conclusions: FNAC proved that it can be a simple, reliable, rapid and safe tool for the preoperative diagnostic procedure for palatal salivary gland lesions. $\mathrm{Co}-\mathrm{Cr}$ needles are superior to Stainless steel needles in FNAC because of its higher biocompatibility.

KEYWORDS: Palatal minor salivary gland tumors, Diagnostic accuracy, Fine needle aspiration biopsy, Cobalt Chromium, Stainless Steel.

* Assistant Professor of Oral and Maxillofacial Surgery, Department of Oral and Maxillofacial Surgery, Tanta University, Tanta, Egypt.

** Lecturer of Oral Pathology, Oral Pathology Department, Tanta University, Tanta, Egypt.

*** Lecturer of Dental Biomaterials, Dental Biomaterials Department, Tanta University, Tanta, Egypt. 


\section{INTRODUCTION}

Salivary gland neoplasms constitute approximately $3-10 \%$ of neoplasms of the head and neck region and have a wide variety of tumors. Salivary gland swellings can be the result of neoplasms, cysts and inflammatory lesions. ${ }^{1-3}$. The general rule in salivary gland neoplasms is: the smaller the gland the higher the rate of malignancy, thus, the rate of malignancy increases from $20 \%-25 \%$ in the parotid gland to $40 \%-50 \%$ in the submandibular gland and to $50 \%-81 \%$ in the sublingual glands and minor salivary glands ${ }^{4-7}$. A proper pre-operative planning is always better than a revision surgery ${ }^{8}$.

Fine Needle Aspiration Cytology (FNAC) has been considered as an inexpensive, minimally invasive, routinely diagnostic method. ${ }^{9}$ FNAC was firstly introduced by "Karolinska Group" 10 . FNAC has been helping clinicians to save their times for earlier diagnosis and treatment. It gives good preservation of cellular details and avoids artifacts which are caused by packing of cells and by cutting knife of microtome in case of paraffin block sections. It is based on the morphological findings of individual cells, group of cells, and micro-particles of tissue ${ }^{11}$.

Aspiration cytology has proved impressive results in almost all lesions everywhere in the body. In salivary glands, FNAC is more effective and easier to perform as the site is quiet superficial. For excellence, cytology provides confirmation of benign diseases and overall surgery can be avoided in nearly one third to one half of the cases ${ }^{12}$. In patients undergoing radical surgery, preoperative FNAC enables the clinician to estimate the degree of urgency and to plan the surgical approach. In case of parotid gland lesions, the decision to preserve or sacrifice the facial nerve is very critical, particularly if adenoid cystic carcinoma is suspicious. The complications of FNAC are rare and tolerable. If any, these may be some bleeding and minimal inflammatory reactions in the region of needle penetration ${ }^{13,14}$.
However, with FNAC, the scarcity of tumor cells in some cases makes the diagnosis a little bit difficult. In addition, there is one more challenge in FNAC which is differentiating benign diseases from malignant ones, then differentiating the various malignancies from one another. ${ }^{16}$

Manufacturers have been making use of a wide range of biomaterials for the fabrication of tools, instruments and devices that can be used in different biological tasks as well as to enable scientists and researchers to carry out biological investigations on different parts of the human body, either to diagnose (biopsy) or to find a suitable treatment for some diseases. An important category of these tools is the needles (attached to syringes) used to collect some tissue fluid or to get some tissue cells from body lesions as a "biopsy".

Needles used to be fabricated from "Stainless Steel" alloy. "Stainless Steel" alloy is characterized by having high strength properties, high hardness and corrosion resistance. Stainless steel was one of the earliest biomaterials used in the human body. Since that time, manufacturers of biomedical materials have been aiming to produce additional alloys with better biomechanical characteristics. ${ }^{17}$ Recently, Cobalt Chromium "Co-Cr" alloy is one of the safest biomaterials to be used in the living tissues because of its promising corrosion properties and mechanical characteristics. The ability to form a passive layer from Chromium oxide " $\mathrm{Cr}_{2} \mathrm{O}_{3}$ " is the main factor behind its excellent corrosion resistance. ${ }^{18,19}$ In addition to its promising corrosion properties, "Co-Cr" alloy has been widely used in biomedical applications because of its superior mechanical properties, wear resistance and biocompatibility. ${ }^{20}$

The primary objective of this study was to examine the reliability of FNAC as a preoperative diagnostic tool for palatal minor salivary gland tumors. In addition, this study investigated and compared the influence - on tissues punctured as 
well as tissues collected - of needles fabricated from Co-Cr alloy with others fabricated from stainless steel alloy in case of aspirating some cells from palatal minor salivary gland tumors for diagnostic purposes.

\section{MATERIALS AND METHODS}

A total of 60 patients with palatal lesions were carefully selected and undergone a preoperative FNAC. Later on, those patients were subjected to histopathological examination as well. This study was conducted in the Departments of Oral Pathology and Oral and Maxillofacial Surgery, Faculty of Dentistry, Tanta University, Tanta, Egypt, over a period from September 2015 to September 2017. FNAC specimens were supplied from selected patients with palatal swellings after obtaining a written medical consent. The experimental protocol for analyzing FNAC and histopathological specimens were reviewed and approved by the Ethical Board of Faculty of Dentistry, Tanta University.

The FNAC was performed using a 22-gauge needle attached to a $10 \mathrm{ml}$ disposable syringes, by a free hand technique, under local anesthesia. Patients were divided into two main groups $(n=30)$; in Group A, the aspiration was done using a CobaltChromium "Co-Cr" needle, attached to a syringe, while in Group B, it was performed using a stainless steel needle. The needle was introduced into the palatal mass and suction applied by withdrawing the syringe plunger to the 1-2 $\mathrm{ml}$ mark. The needle was moved backward \& forward four to five times in the same plane to ensure minimal bleeding. Thin smears were prepared between two slides and immediately fixed with $90 \%$ alcohol while being wet then stained with "Hematoxylin and Eosin" (H\&E) stain.

The biopsy (either incisional or excisional) was immediately taken after aspiration and the surgical specimens were fixed in $10 \%$ formalin, then routinely processed and stained by (H\&E) stain for histopathological examinations. The aspiration cytology was reported by a single consultant cytopathologist who was not aware of the histopathological examination report.

Data were collected and analyzed to determine the overall accuracy of FNAC to differentiate between benign and malignant diseases.

\section{RESULTS}

Sixty patients with palpable palatal lesions were included in this study. The age group maximally affected were from 20-29 years (24.28\%). Among these 60 patients; 36 were males and 24 were females (male: female 1.5: 1).

\section{Cytopathological results}

In this study, 45 specimens were benign (pleomorphic adenomas, $75 \%$ ) and 7 specimens were malignant (5 mucoepidermoid carcinomas and 2 adenoid cystic carcinomas, $11.67 \%$ ). The remaining 8 specimens revealed squamous cell carcinomas, non-specific inflammation and normal salivary gland tissue (Table 1).

For pleomorphic adenoma that was characterized by numerous ductal structures and highly cellular component with plasmacytoid appearance (Fig. 1a, b). Cytologically, there were cellular glandular cells with characteristic fibrillar appearance of the matrix (Fig 1c, d) (H \& E stain, 400×).

In mucoepidermoid carcinoma with both mucous secreting cells and epidermoid cells, there were large single mucous secreting cells floating in association with other smaller cells with hyperchromatic nuclei. In case of adenoid cystic carcinoma with characteristic cribriform pattern, there were small clusters of abnormal glandular isomorphic cells with hyperchromatic nuclei and coarse chromatin.

\section{Histopathological results}

H\&E - stained sections of excised lesions revealed 43 benign (Pleomorphic adenomas, 


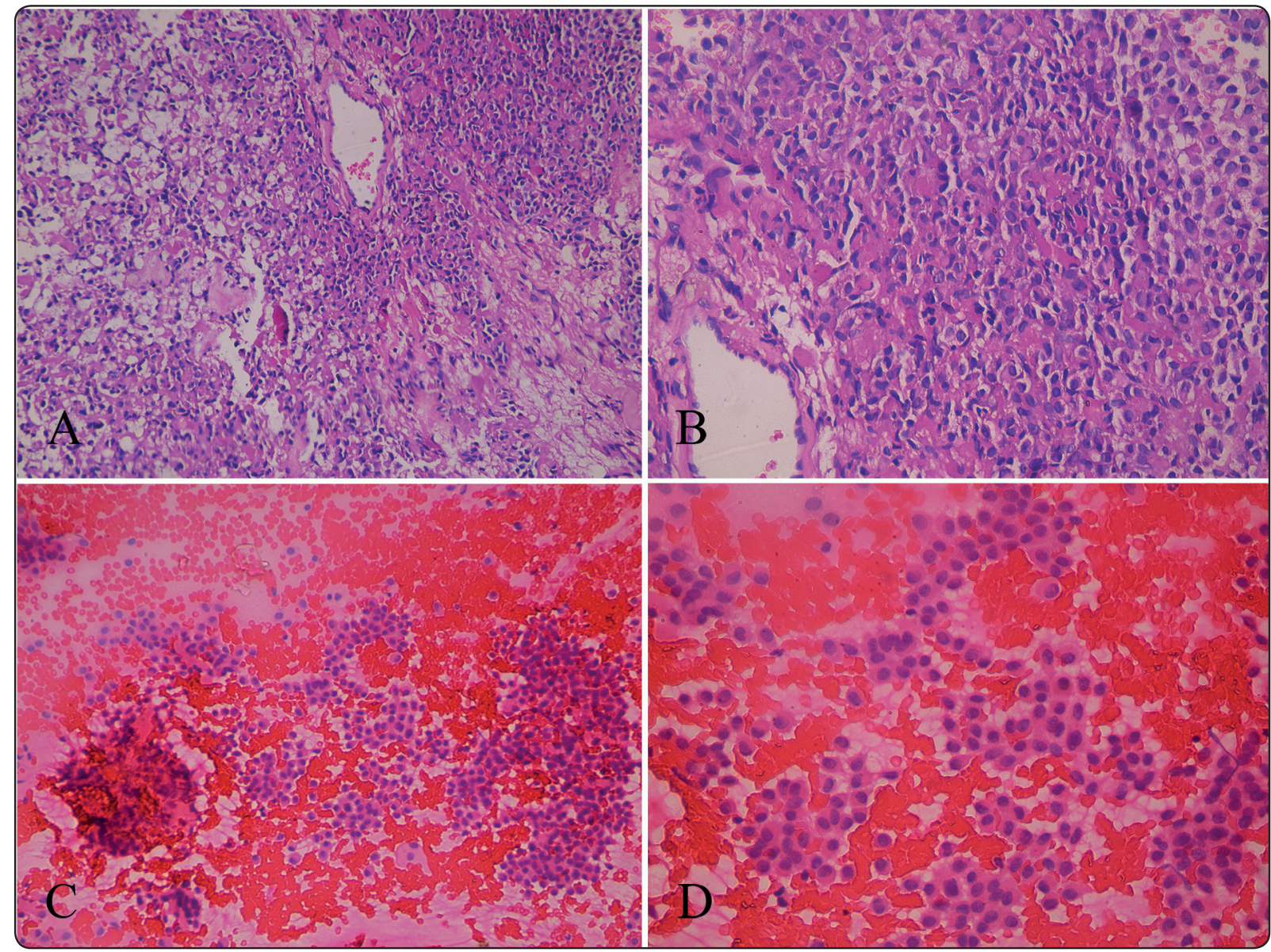

Fig. (1) FNAC of pleomorphic adenoma. Histopathologically, pleomorphic adenoma was characterized by numerous ductal structures and highly cellular component with plasmacytoid appearance (Fig. 1A, B). Cytologically, there were cellular glandular cells with characteristic fibrillar appearance of the matrix (Fig 1C, D) (H \& E stain, 400×).

$71.67 \%)$ and 14 malignant (8 mucoepidermoid carcinomas, 3 adenoid cystic carcinomas and 3 squamous cell carcinomas, $23.33 \%$ ) lesions were diagnosed. The histopathologic examination of the remaining lesions revealed 2 chronic sialadenitis and one hyperplastic minor salivary gland tissue (Table 1). Cytological and histopathological diagnoses were matching in 56 specimens (93.39\% of cases).

\section{Surgical management of cases}

The surgical treatment was performed according to the matching between the cytopathological and histopathological diagnoses. The cases that were diagnosed pleomorphic adenoma were surgically excised without safety margins (Fig. 2a-d). However, in cases of malignant lesions, they were planned for further investigations before surgery.

\section{Effect of needle material on the quality of collected cells}

The cytological examination revealed reduced inflammation, hemorrhage and minimal deterioration in the collected cells in case of "Co-Cr" needles compared to those collected by "Stainless Steel" needles. Also, clinical follow up of the lesions revealed minimal post-operative infection in those punctured with "Co-Cr" needles compared to "Stainless Steel" needles. 

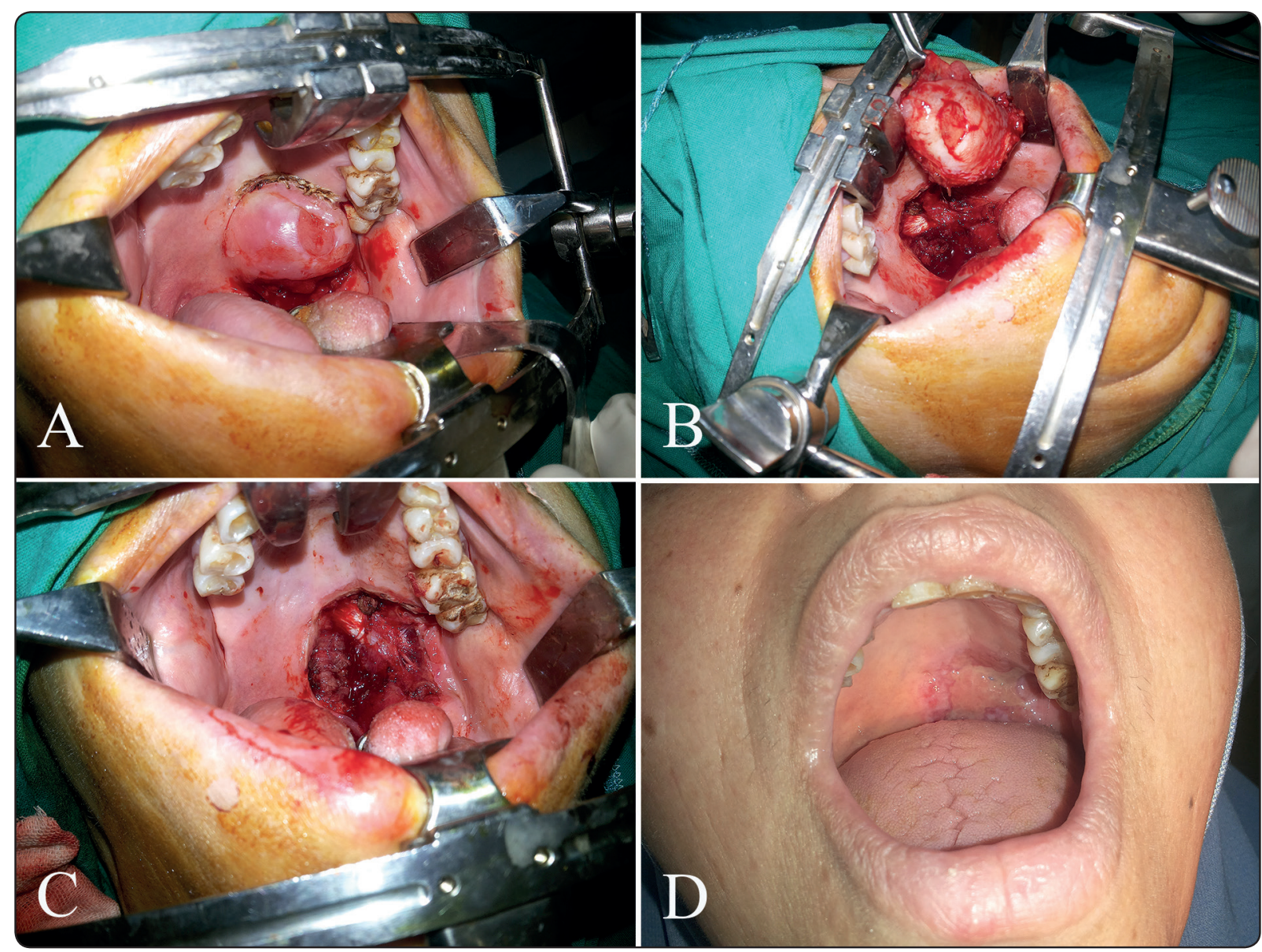

Fig. (2) Surgical treatment was performed according to the matching between the cytopathological and histopathological diagnoses. Pleomorphic adenoma were surgically excised without safety margins (Fig. 2A-D).

\section{DISCUSSION}

Although some authors considered the FNAC as a superior modality and claimed its accuracy and safety, others argued that it has little influence on clinical management because of its high rates of false-positives and false-negatives and patients ultimately have to undergo surgery ${ }^{4,9}$.

Palatal swellings remain a diagnostic challenge among oral surgeons. For many years, biopsy has been the established diagnostic procedure to confirm the diagnosis of salivary gland tumors. But it takes nearly longer time to get the histopathology report whereas FNAC report can be obtained within two days. Thus, this study was performed to explain the potency of FNAC as an alternative and reliable diagnostic procedure. FNAC provides a suitable method to obtain a tissue for diagnosis and therefore became a considerable diagnostic test to solve the dilemma of palatal swellings.

The results of this study confirmed the accuracy and clinical utility of FNAC in the diagnosis of benign and malignant salivary gland tumors. The diagnostic accuracy of FNAC in our study was similar to those in various other studies.

The total number of suspected salivary gland neoplasms was low within the period from 
September 2015 to September 2017. We excluded the palatal abscesses and other fluctuant cystic lesions to have our study focusing on salivary gland lesions. Non-neoplastic lesions such as chronic sialadenitis were included as they appeared as palatal masses.

Pleomorphic adenoma was the most common lesion $(71.67 \%)$ within the 60 selected patients. Majority of such tumors involved the posterior part of the hard palate, with $95.5 \%$ diagnostic accuracy. In the parotid gland, pleomorphic adenomas account for more than $70 \%$ of all the tumors, but they rarely occur in the sublingual gland..$^{21}$ Cytologic smears revealed three main components: fibrillar matrix, myoepithelial and ductal cells in various proportions. ${ }^{21}$ Diagnosis of a pleomorphic adenoma is confirmed after the presence of the three components previously described. When stromal effects are minimal or high, cellular cases are difficult to diagnose as pleomorphic adenomas and they may be confused with other tumors of the salivary gland. ${ }^{22,23}$ Because of the varied, complex and overlapping morphological features, the diagnosis of pleomorphic and adenoid cystic carcinoma can be sometimes problematic.

Mucoepidermoid carcinoma (MEC) and adenoid cystic carcinoma were the only malignant salivary gland lesions seen in our study. Ineffective diagnosis of low grade MEC is a common problem, because of bland cytological features and clear cells which resemble macrophages. MECs can occur at any age, they can involve both the major and minor salivary glands, and they are the most common malignant salivary gland tumors affecting children. ${ }^{24}$

The most important question to be answered by our study was whether results gained by FNAC can be helpful in the clinical management of patients with palatal masses. Our experience has demonstrated a variety of circumstances in which such data may be valuable. The usual recommendation for the neoplastic lesions regardless of the preoperative cytological diagnosis is excision but recognition of benign lesion in poor risk patients may be of benefit in avoiding inappropriate surgery.

The accuracy of FNAC exhibit a wide variation both in published literature as well as in the community. ${ }^{25}$ Proper training and ability in specimen interpretation is essential. ${ }^{26}$ Studies have shown that the majority of missed diagnosis is due to problems in sampling and specimen preparation. ${ }^{27,28}$ In the current study, the sampling was quiet adequate in all cases.

The only relative contraindication for FNAC could be a disease with bleeding tendency. Many authors exclude the possibility of the implantation of the malignant cells or its recurrence caused by FNAC. ${ }^{29}$ The phenomenon of tumor cells seeding has become a rare complication with the current use of small-bored needles. ${ }^{30}$

With regard to the material of the needles used in this study, the main property required for a biomaterial is that it does not produce or initiate an adverse reaction when placed into a living tissue, that is, to be a biocompatible material. Excellent corrosion resistance, good mechanical properties and enhanced wear resistance are crucial requirements of such a material as well. ${ }^{31}$

The absence of or minimal tissue reaction to the needle penetration in case of "Co-Cr" alloy compared to that in case of "Stainless Steel" needle can be interpreted on the basis that "Co-Cr" alloy has higher biocompatibility than "Stainless Steel" alloy. It has been reported that "Co-Cr" alloys are well-tolerated by tissue cells with no significant side effects. ${ }^{32}$

In conclusion, with the close matching between FNAC and histological results, FNAC proved to be easy, non-traumatic, economic, safe and reliable for preoperative diagnosis of salivary gland tumors and in the differentiation of benign and malignant salivary gland tumors in the palatal region. A preoperative malignant diagnosis allows the oral surgeons to plan the treatment, while a benign diagnosis relieves the patient from anxiety and 
surgical procedures in cancer phobia - patients. Preoperative differentiation of tumors may help prepare both the surgeon and patient for an appropriate surgical procedure. In addition, $\mathrm{Co}-\mathrm{Cr}$ needle proved better biocompatibility with tissues punctured and lower deformity of cells collected than did stainless steel needles.

\section{REFERENCES}

1. Young N A, Mody D R, Davey D D. Misinterpretation of normal cellular elements in fine-needle aspiration biopsy specimens: bservations from the College of American Pathologists Interlaboratory Comparison Program in Non-Gynecologic Cytopathology. Arch Pathol Lab Med 2002;126: 670-5.

2. Henry-Stanley M J, Beneke J, Bardales R H, Stanley M W.Fine-needle aspiration of normal tissue from enlarged salivary glands: sialosis or missed target? Diagn Cytopathol 1995; 13: 300-30.

3. Boccato P, Altavilla G, Blandamura S. Fine needle aspiration biopsy of salivary gland lesions. A reappraisal of pitfalls and problems. Acta Cytol 1998; 42: 888-98.

4. Batsakis JG, Sneige N, and El-Naggar AK, "Fine needle aspiration in salivary glands: its utility and tissue effects," Annals of Otology, Rhinology and Laryngology 1992; 101:185-188.

5. Freling NJ, Molenaar WM, Vermey A, et al. Malignant parotid tumors: clinical use of MR imaging and histologic correlation. Radiology 1992; 185:691-6.

6. Weber RS, Byers RM, Petit B, Wolf P, Ang K, Luna M.Submandibular gland tumors: adverse histologic factors and therapeutic implications. Arch Otolaryngol Head Neck Surg 1990; 116:1055-60.

7. Shah JP. Head and neck surgery. 2nd ed. London, United Kingdom: Mosby-Wolfe, 1996;431-60.

8. Chilla R. Revision of the parotid-assessing the risk and indications status for re-parotidectomy.HNO. 1990 Jan;38(1):33-7.

9. Orell SR, Sterrett GF, Walters MN-I, Whitaker D: Fine Needle Aspiration Cytology. New York: Churchill Livingstone; 1992.P. 7-23.-

10. Fernandes GC, Pandit AA. Diagnosis of salivary gland tumours by FNAC. Bombay Hospital Journal 2000; 42:108-11.
11. Kamal MM, Dani AA, Kotwal MN, Kherdekar MS. Aspiration cytology of salivary gland lesions advantages and pitfalls. Indian J Pathol Microbiol 1994; 37:281-7.

12. Nettle WJS, Orell SR. Fine needle aspiration cytology in the diagnosis of salivary gland lesions. Aust NZ J Surg 1989; 59: 47-51.

13. Zbaren P, Nuyens M, Loosli H, Stauffer E. Diagnostic accuracy of fine-needle aspiration cytology and frozen sections in primary parotid carcinoma. Cancer 2004; 100: 1876-83.

14. Stewart CJ, MacKenzie K, McGarry GW, Mowat A. Fineneedle aspiration cytology of the salivary gland: a review of 341 cases. Diagn Cytopathol 2000; 22:139-46.

15. Fulciniti F, Califano L, Zupi A, Vetrani A. Accuracy of fine needle aspiration biopsy in head and neck tumours. J Oral Maxillofac Surg. 1997;55:1094-7.

16. Vaidya S, Sinha A, Narayan S, Adhikari S and KC Sabira1: A comparative study of fine-needle aspiration cytology and histopathology in salivary gland lesions, Journal of Pathology of Nepal (2011) Vol. 1, 108 -113

17. Bhat, Sujata V., Biomaterials (2nd ed.). Harrow, UK: Alpha Science International Ltd. (2005).

18. Lewandowska-Szumiel M, Komender J, Chlopek J. Cobalt-based alloy. J Biomed. Mater. Res. 48: 289 (1999).

19. Fujihara K, Huang Z M, Ramakrishna S, Satknanantham K, Hamada H, "Biomaterials". 24: 2661 (2003).

20. Granchi D, Ciapetti G, Stea S, Savarino L, Filippini F, Sudanese A, Zinghi and Montanaro L: Biomaterials 20 (1999) 1079-1086.

21. Mukunyadzi P. Review of Fine-Needle Aspiration Cytology of Salivary Gland Neoplasms, With Emphasis on Differential Diagnosis. Am J Clin Pathol. 02; 118 (Suppl 1): S100-S115S.

22. Stanley MW, Lowhagen T. Mucin production by pleomorphic adenomas of the parotid gland: a cytologic spectrum. Diagn Cytopathol. 1990; 6:49-52100.

23. Handa U, Dhingra N, Chopra R, Mohan H. Pleomorphic Adenoma: Cytologic variations and potential pitfalls. Dign Cytopathol. 37:12-15.

24. Stanley MW. Head and neck cytology. In: Silverberg SG, ed.Principles and Practice of Surgical Pathology and Cytopathology.Vol 2. 3rd ed. New York, NY: Churchill Livingstone. 1997; 995-1037. 
25. Giard RW, Hermans J. The value of aspiration cytologic examination of the breast: a statistical review of the medical literature. Cancer. 1992, 69:2104-2110.

26. Zarbo RJ, Howanitz PJ, Bachner P. Interinstitutional comparison of performance in breast fine needle aspiration cytology: a Q - probe quality indicator study. Arch Pathol Lab Med. 1991, 115:743-750

27. Lee KR, Foster RS, Papillo JI. Fine needle aspiration of breast: importance of the aspirator. Acta Cytol. 1987,31:281-284.

28. Cohen MB, Rodgers C, Hales MS et al. The importance of training and experience in the interpretation of fine needle aspirates of the breast: analysis by ROC curves. Arch Pathol LabMed. 1987, 111:518-520.
29. Glant MD, "Cytopathology of lymph nodes in nonspecific reactive hyperplasia. Prognostication and differential diagnoses," American Journal of Clinical Pathology 1997; 108: S31-S55.

30. R.G. Amedee and N.R. Dhurandhar, "Fine-needle aspiration biopsy," Laryngoscope 2001; 111: 1551-1557.

31. Black, J. \& Hastings G.W. Handbook of biomaterials properties. London UK: Chapman and Hall; 1998.

32. Granchi, D., Ciapetti, G., Savarino, L., Cavedagna, D., Donati, M. E., and Pizzoferrato, A. (1996). Assessment of metal extract toxicity on human lymphocytes cultured in vitro. J. Biomed. Mater. Res. 31, 183-191. 